\title{
What's hiding in the PDB? Reinvestigation of structural data for the biomedically important enzymes metallo- $\beta$-lactamases
}

\author{
Joanna E. Raczynska, Ivan G. Shabalin, Alexander Wlodawer, Wladek Minor, Mariusz Jaskolski
}

Antibiotic resistance is a serious and steadily growing medical threat. One possible mechanism of resistance is the expression of $\beta$-lactamases, which have the ability to hydrolyze $\beta$ lactam antibiotics. The most worrisome group of these enzymes are metallo- $\beta$-lactamases (MBLs) which employ catalytic $\mathrm{Zn}$ ions to perform the hydrolysis reaction. Mechanistically they are different from other $\beta$-lactamases (such as serine hydrolases) and they are resistant to the clinically available $\beta$-lactamase inhibitors. MBLs have been extensively studied. Structurally they exhibit a considerable diversity of their binding sites and substrate specificities, making inhibitor design difficult. Unfortunately, a high level of noise in the structural information, in particular in the aspect of enzyme-ligand binding, makes it difficult to carry out detailed comparisons among structures, not to mention drawing of meaningful biological or medical conclusions from such comparisons.

We have analyzed all of the available MBL structures in the PDB in terms of various quality criteria, with special focus on the bound ligands, their electron density fit and interactions. We found some serious inconsistencies between the published crystallographic models and the supporting experimental data. The problems include ligands that are completely or partially absent, ligands that are in fact different compounds than what is claimed in the publications, or ligands presented as novel reaction intermediates without any supporting crystallographic evidence.

In several cases we have re-refined the structural models against available structure factors and were able to obtain significant improvement of $\mathrm{R} / \mathrm{R}_{\text {free }}$ and other quality criteria. Thanks to the improved phases, we were able to better model the important parts of those structures and sometimes apply significant corrections to the key elements of the molecules. Here we present the most interesting cases of the re-analysis and highlight the significance of our findings.

J oanna Raczynska Ivan Shabalin

Alexander Wlodawer Wladek Minor

Mariusz J askolski
Poland Institute of Bioorganic Chemistry, Polish Academy of Sciences, Poznan

USA Department of Molecular Physiology and Biological Physics, University of Virginia, Charlottesville USA Macromolecular Crystallography Laboratory, National Cancer Institute at Frederick

USA Department of Molecular Physiology and Biological Physics, University of Virginia, Charlottesville Poland Department of Crystallography, Faculty of Chemistry, A. Mickiewicz University, Poznan 